Ferrata Storti Foundation

\title{
Once-weekly versus twice-weekly carfilzomib in patients with newly diagnosed multiple myeloma: a pooled analysis of two phase I/II studies
}

Haematologica 2019

Volume 104(8):1640-1647

\section{Correspondence:}

SARA BRINGHEN

sarabringhen@yahoo.com

Received: October 4, 2018.

Accepted: February 7, 2019.

Pre-published: February 7, 2019.

doi:10.3324/haematol.2018.208272

Check the online version for the most updated information on this article, online supplements, and information on authorship \& disclosures: www.haematologica.org/content/104/8/1640

\section{(C)2019 Ferrata Storti Foundation}

Material published in Haematologica is covered by copyright. All rights are reserved to the Ferrata Storti Foundation. Use of published material is allowed under the following terms and conditions:

https://creativecommons.org/licenses/by-nc/4.0/legalcode. Copies of published material are allowed for personal or internal use. Sharing published material for non-commercial purposes is subject to the following conditions:

https://creativecommons.org/licenses/by-nc/4.0/legalcode, sect. 3. Reproducing and sharing published material for commercial purposes is not allowed without permission in writing from the publisher.

\section{(c) (i) (s)}

\section{Sara Bringhen, ${ }^{1}$ Roberto Mina, ${ }^{1}$ Maria Teresa Petrucci, ${ }^{2}$ Gianluca Gaidano,${ }^{3}$ Stelvio Ballanti, ${ }^{4}$ Pellegrino Musto, ${ }^{5}$ Massimo Offidani, ${ }^{6}$ Stefano Spada, ${ }^{1}$ Giulia Benevolo, ${ }^{7}$ Elena Ponticelli, ${ }^{1}$ Piero Galieni, ${ }^{8}$ Michele Cavo, ${ }^{9}$ Tommaso Caravita Di Toritto, ${ }^{10^{*}}$ Francesco Di Raimondo, ${ }^{11}$ Vittorio Montefusco, ${ }^{12}$ Antonio Palumbo, ${ }^{1+*}$ Mario Boccadoro ${ }^{1}$ and Alessandra Larocca ${ }^{1}$}

\begin{abstract}
${ }^{1}$ Myeloma Unit, Division of Hematology, University of Torino, Azienda OspedalieroUniversitaria Città della Salute e della Scienza di Torino, Torino; '2Division of Hematology, Department of Cellular Biotechnologies and Hematology, Sapienza University of Rome, Rome; ${ }^{3}$ Division of Hematology, Department of Translational Medicine, Università del Piemonte Orientale, Novara; ${ }^{4}$ Sezione di Ematologia e Immunologia Clinica, Ospedale Santa Maria della Misericordia di Perugia, Perugia; ${ }^{5}$ Unit of Haematology and Stem Cell Transplantation, IRCCS-CROB, Referral Cancer Center of Basilicata, Rionero in Vulture; ${ }^{6}$ Clinica di Ematologia, AOU Ospedali Riuniti di Ancona, Ancona; ${ }^{7}$ Hematology, Città della Salute e della Scienza, Turin; ${ }^{8}$ Division of Hematology, Ospedale “C. e G. Mazzoni”, ASUR Marche-AV5, Ascoli Piceno; "'Seràgnoli" Institute of Hematology, Bologna University School of Medicine, Bologna; ${ }^{10}$ UOC Ematologia, Ospedale S. Eugenio, ASLRM2, Rome; ${ }^{11}$ Division of Hematology, AOU Policlinico-OVE, University of Catania, Catania and ${ }^{12} \mathrm{Hematology}$ Department, Fondazione IRCCS Istituto Nazionale Tumori, Milano, Italy
\end{abstract}

${ }^{*}$ currently employed at UOSD Ematologia, P.O. S. Spirito e Nuovo Regina Margherita ASLRM1, Rome;

${ }^{*}$ currently employed at Takeda Pharmaceuticals International, Switzerland

\section{ABSTRACT}

T wice-weekly carfilzomib is approved at 27 and $56 \mathrm{mg} / \mathrm{m}^{2}$ to treat relapsed multiple myeloma patients. In the phase III study ARROW, once-weekly $70 \mathrm{mg} / \mathrm{m}^{2}$ carfilzomib prolonged the median progression-free survival of relapsed multiple myeloma patients in comparison with twice-weekly $27 \mathrm{mg} / \mathrm{m}^{2}$ carfilzomib, without adding significant toxicity. Data were pooled from two phase I/II studies of newly diagnosed multiple myeloma patients who received nine induction cycles of carfilzomib (either $70 \mathrm{mg} / \mathrm{m}^{2}$ once-weekly or $36 \mathrm{mg} / \mathrm{m}^{2}$ twice-weekly), cyclophosphamide and dexamethasone, followed by carfilzomib maintenance. Overall, 121 transplant-ineligible patients with newly diagnosed multiple myeloma were analyzed (once-weekly, $n=63$; twice-weekly, $n=58$ ). We found no significant difference in median progression-free survival [35.7 months (95\% CI: 23.7-not reached, NR) vs. 35.5 months (95\%CI: 24.3-NR); HR: 1.39; $P=0.26$ and 3 -year overall survival [70\% [95\%CI: 59\%-84\%) vs. 72\% (95\%CI: $60 \%$ $85 \%)$; HR: $1.27 ; P=0.5]$ between once-weekly and twice-weekly carfilzomib. From the start of maintenance, 3-year progression-free survival $[47 \%$ (95\%CI: 33\%-68\%) vs. 51\% (95\%CI: 38\%-70\%); HR: $1.04 ; P=0.92]$ and overall survival [72\% (95\% CI: 58\%-89\%) vs. 73\% (95\% CI: 59\%-90\%); HR: $0.82 ; P=0.71]$ were similar in the once- versus twice-weekly carfilzomib. The rate of grade $3-5$ hematologic $(24 \%$ vs. $30 \% ; P=0.82)$ and non-hematologic ( $38 \%$ vs. $41 \% ; P=0.83$ ) adverse events was similar in the two groups. Onceweekly $70 \mathrm{mg} / \mathrm{m}^{2}$ carfilzomib as induction and maintenance therapy for newly diagnosed multiple myeloma patients was as safe and effective as twice-weekly $36 \mathrm{mg} / \mathrm{m}^{2}$ carfilzomib and provided a more convenient schedule. The trials are registered at clinicaltrials.gov identifiers: 01857115 (IST-CAR561) and 01346787 (IST-CAR-506). 


\section{Introduction}

In the last two decades, several novel agents of various classes have been developed and approved to treat multiple myeloma (MM), resulting in improved overall survival (OS) for both transplant-eligible and -ineligible patients. ${ }^{1}$ Among new agents, the immunomodulatory drugs (IMiD) thalidomide and lenalidomide, and the proteasome inhibitor (PI) bortezomib, have been included in the initial treatment for newly diagnosed (ND) MM patients. Bortezomib, a first-generation PI, proved to be a very effective anti-MM agent. It was initially approved for the relapse setting and then approved for upfront therapy. Despite the efficacy of bortezomib, its long-term administration is limited by the emergence of peripheral neuropathy, which was reported in $4-13 \%$ of patients (grade 3-4).,2,3

Carfilzomib, a second-generation PI, showed significant activity among patients with relapsed and/or refractory (RR) MM and was approved by US Food and Drug Administration and the European Medicines Agency in combination with dexamethasone or lenalidomide-dexamethasone $(\mathrm{Rd})$ for the treatment of RRMM patients. Given the efficacy displayed by carfilzomib in the relapse setting, several trials tested carfilzomib as part of upfront therapy for NDMM patients, either with Rd (KRd) or with alkylating agents, such as melphalan-prednisone (KMP) or cyclophosphamide-dexamethasone (KCyd) ${ }^{4 \cdot 7}$

Carfilzomib is currently approved with the twice-weekly schedule at a dose of $27 \mathrm{mg} / \mathrm{m}^{2}$ over a 2-10-minute $(\mathrm{min})$ infusion period when administered alone or in combination with lenalidomide and dexamethasone, or at a dose of $56 \mathrm{mg} / \mathrm{m}^{2}$ over a 30 -min infusion period when given in combination with dexamethasone (Kd).
Nonetheless, other doses (up to $70 \mathrm{mg} / \mathrm{m}^{2}$ ) and schedules (once weekly) have been shown to be promising.

The current twice-weekly schedule may not be very convenient for patients (particularly for elderly patients with limited access to hospital facilities), affecting their quality of life and treatment compliance. In order to improve the convenience of the carfilzomib schedule, preliminary studies tested higher doses of carfilzomib administered in a once-weekly schedule. The phase Ib/II CHAMPION-1 study tested different doses of once-weekly carfilzomib in RRMM patients to define its maximum tolerated dose (MTD) combined with dexamethasone. ${ }^{8}$ The MTD of once-weekly carfilzomib proved to be 70 $\mathrm{mg} / \mathrm{m}^{2}$ over a 30-min infusion period, displaying good efficacy and tolerability. Based on these results, a phase III study (ARROW) was initiated to compare twice-weekly carfilzomib at the dose of $27 \mathrm{mg} / \mathrm{m}^{2}$ with once-weekly carfilzomib at the dose of $70 \mathrm{mg} / \mathrm{m}^{2} .{ }^{9}$ Among $578 \mathrm{RRMM}$ patients, once-weekly carfilzomib improved the overall response rate (ORR; $62.9 \%$ vs. $40.8 \%$ ) and prolonged median progression-free survival (PFS) as compared to twice-weekly carfilzomib (median PFS, 11.2 vs. 7.6 months), with a similar rate of grade 3-4 adverse events (68\% vs. $62 \%$ ). A major limitation of the ARROW study was the low dose $\left(27 \mathrm{mg} / \mathrm{m}^{2}\right)$ of carfilzomib in the twiceweekly arm as compared to the $70 \mathrm{mg} / \mathrm{m}^{2}$ dose adopted in the once-weekly arm. This low dose was determined according to the carfilzomib approval at the time of study design.

We previously published data from two phase I/II (ISTCAR-561) and phase II (IST-CAR-506) studies investigating once-weekly $\left(70 \mathrm{mg} / \mathrm{m}^{2}\right)$ and twice-weekly $\left(36 \mathrm{mg} / \mathrm{m}^{2}\right)$ carfilzomib combined with cyclophosphamide and dex-

Table 1. Patients' characteristics.

\begin{tabular}{|c|c|c|c|c|}
\hline & $\begin{array}{c}\text { All patients } \\
\mathrm{N}=121\end{array}$ & $\begin{array}{c}\begin{array}{l}\text { IST-CAR-561 } \\
\text { Once-weekly } \\
N=63\end{array}\end{array}$ & $\begin{array}{c}\text { IST-CAR-506 } \\
\text { Twice-weekly } \\
\text { N=58 }\end{array}$ & $P$ \\
\hline Age, median (range) & $72(55-86)$ & $72(60-85)$ & $71(55-86)$ & 0.67 \\
\hline$\geq 75$ years & $31(26 \%)$ & $14(22 \%)$ & $17(29 \%)$ & 0.41 \\
\hline Sex, female & $68(56 \%)$ & $37(59 \%)$ & $31(53 \%)$ & 0.59 \\
\hline $\begin{array}{l}\text { Serum creatinine, mg/dL, } \\
\text { median (range) }\end{array}$ & $0.90(0.46-3.7)$ & $0.82(0.5-3.7)$ & $1.00(0.46-2.92)$ & 0.06 \\
\hline \multicolumn{5}{|l|}{ ISS } \\
\hline 1 & $40(33 \%)$ & $24(38 \%)$ & $16(28 \%)$ & 0.46 \\
\hline 2 & $38(31 \%)$ & $19(30 \%)$ & $19(33 \%)$ & \\
\hline 3 & $43(36 \%)$ & $20(32 \%)$ & $23(40 \%)$ & \\
\hline \multicolumn{5}{|l|}{ FISH } \\
\hline Standard risk & $57(47 \%)$ & $24(38 \%)$ & $33(57 \%)$ & 0.4 \\
\hline High risk & $37(31 \%)$ & $19(30 \%)$ & $18(31 \%)$ & \\
\hline Missing & $27(22 \%)$ & $20(32 \%)$ & $7(12 \%)$ & \\
\hline \multicolumn{5}{|l|}{ Frailty Score } \\
\hline Fit & $67(55 \%)$ & $37(59 \%)$ & $30(52 \%)$ & 0.19 \\
\hline Intermediate fitness & $40(33 \%)$ & $22(35 \%)$ & $18(31 \%)$ & \\
\hline Frail & $14(12 \%)$ & $4(6 \%)$ & $10(17 \%)$ & \\
\hline LDH, [UI/mol] median (range) & $296(81-768)$ & $306(100-768)$ & $278(81-654)$ & 0.35 \\
\hline Missing & $20(17 \%)$ & $2(3 \%)$ & $18(31 \%)$ & \\
\hline
\end{tabular}

N: number; IQR: interquartile range; LDH: lactate dehydrogenase; ISS: International Staging System; FISH: fluorescence in situ hybridization. 
amethasone (KCyd) as initial treatment for transplantineligible NDMM patients. ${ }^{6,7}$ In both trials, KCyd was shown to be a safe and effective option for NDMM patients. Here we report the results of a pooled analysis of these two studies.

\section{Methods}

\section{Study design and participants}

For this analysis, we pooled together data from two phase I/II (IST-CAR-561; clinicaltrials.gov identifier: 01857115) and phase II (IST-CAR 506; clinicaltrials.gov identifier: 01346787) studies; these studies were led by the same co-operative groups. Patients were recruited from 14 sites across Italy (hospitals, clinics, oncology or medical centers). Both trials enrolled NDMM patients older than 65 years of age or younger but not eligible for autologous stem-cell transplantation. Inclusion and exclusion criteria are similar between the two source studies and have been previously published. ${ }^{6,7}$ Ethics committees or institutional review boards at the study sites approved both studies, which were carried out in accordance with the Declaration of Helsinki. All patients provided written informed consent.

\section{Procedures}

In both studies, patients received nine 4-week induction cycles with carfilzomib, cyclophosphamide (orally, $300 \mathrm{mg}$ on days 1, 8 and 15) and dexamethasone (40 mg on days 1, 8, 15 and 22). In the IST-CAR 561 study, patients received once-weekly carfilzomib at the dose of $70 \mathrm{mg} / \mathrm{m}^{2}$ (on days 1, 8 and 15), while in the IST-CAR 506 study patients received twice-weekly carfilzomib at the dose of $36 \mathrm{mg} / \mathrm{m}^{2}$ (on days 1, 2, 8, 9, 15 and 16). After the induction phase, patients received maintenance treatment with carfilzomib as single agent, which was administered at the same dose and schedule of the induction phase and until progressive disease or intolerable toxicity. Details of study procedures have been previously published. ${ }^{6,7}$

\section{Outcomes}

Focusing on patients who received once-versus twice-weekly

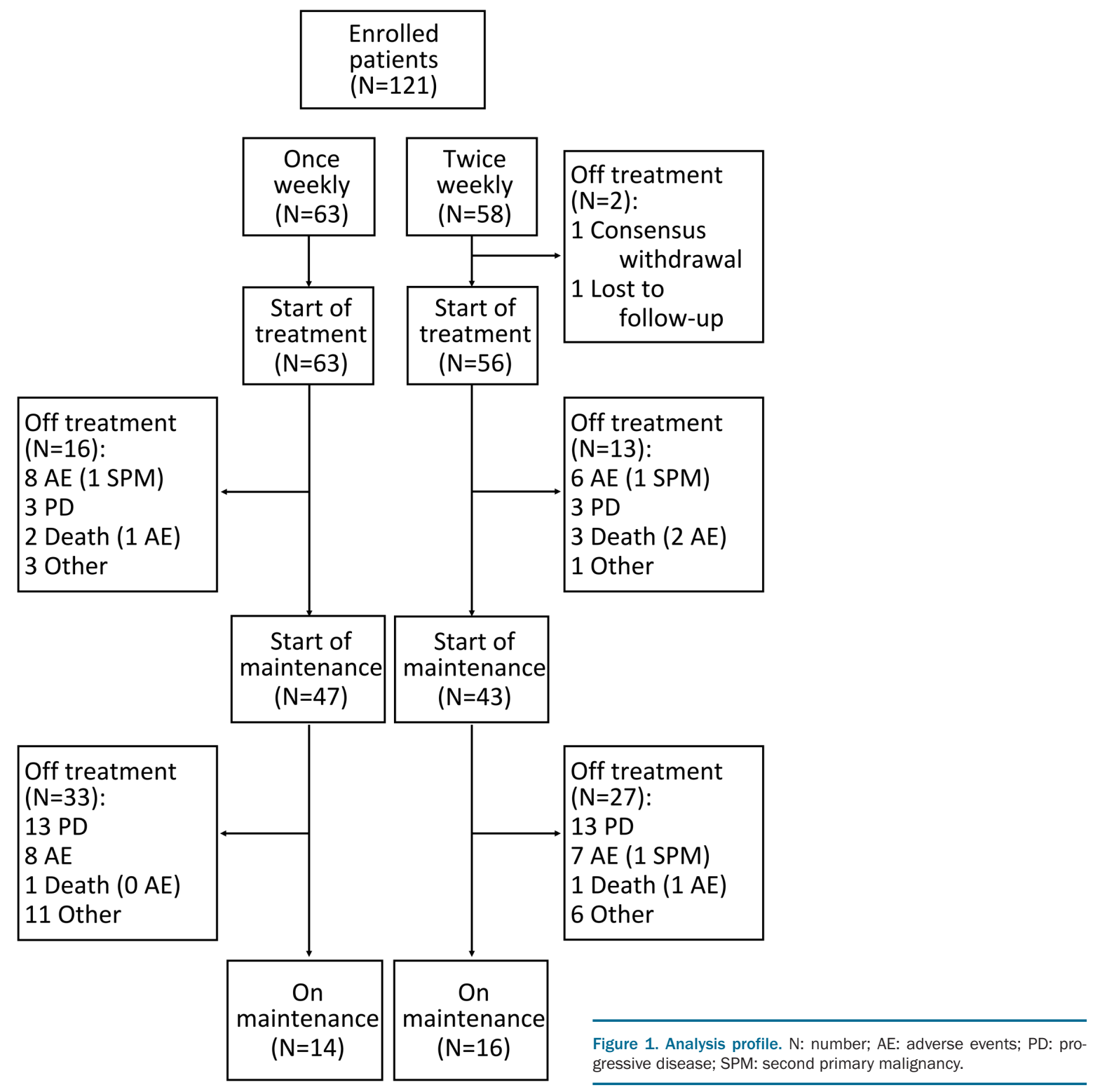


carfilzomib, the primary goals of this analysis were: (1) to compare PFS, PFS-2 and OS from the date of entry onto the trial in the intention-to-treat (ITT) population; (2) to compare PFS from start of maintenance therapy (PFS_m), PFS 2 from start of maintenance therapy (PFS-2_m) and overall survival from start of maintenance therapy (OS_m) in a population who completed the induction phase and started maintenance treatment. (Note that PFS-2 was calculated from the date of enrollment to the date of second relapse/progression or death or the date the patient was last known to be in remission.)

Secondary end points were responses, time to response, and safety of once- versus twice-weekly carfilzomib.

Responses were recorded at the beginning of every cycle, according to the International Myeloma Working Group (IMWG) criteria. All adverse events (AE) were assessed during each cycle and graded according to the National Cancer Institute's Common Terminology Criteria for Adverse Events (version 4.0). ${ }^{10}$ Fluorescence in situ hybridization (FISH) was centrally assessed with a $10 \%$ cut-off for numerical aberrations and a $15 \%$ cut-off for IgH translocations; high-risk FISH was defined by the presence of at least one of the following chromosomal abnormalities: del(17p), t(4;14) or $\mathrm{t}(14 ; 16) .{ }^{11}$ Frailty status was evaluated according to the IMWG Frailty Score. ${ }^{12}$

The intention-to-treat population consisted of all the enrolled patients and was the basis for the analysis of efficacy end points. Patients were analyzed according to initial treatment assignment. The safety population was defined as all the enrolled patients who received at least one dose of carfilzomib, cyclophosphamide or dexamethasone, and was the basis for the analysis of the safety end points.

\section{Statistical analysis}

Data from the two studies were pooled together and analyzed. Comparisons between different patient groups were investigated using Fisher exact test. Time to response was calculated from the start of treatment to the date of the first response [complete remission (CR), partial remission (PR)]. PFS was calculated from the date of enrollment to the date of progression or death or the date the patient was last known to be in remission. PFS- 2 was calculated from the date of enrollment to the date of second relapse/progression or death or the date the patient was last known to be in remission. OS was calculated from the date of enrollment to the date of death or the date the patient was last known to be alive. PFS_m, PFS-2_m and OS_m were calculated from the date of the start of maintenance therapy. In order to account for potential confounders, the comparison once- versus twice-weekly carfilzomib was adjusted for age, International Staging System (ISS), FISH, Frailty Score, and, in relation to the maintenance analysis, for response to induction therapy.

Time-to-event data were analyzed using the Kaplan-Meier method; survival curves were compared with the log-rank test. Results are presented as hazard ratios (HRs), 95\% confidence intervals (95\% CIs), and two-sided $P$-values. Data were censored on September $30^{\text {th }} 2015$ for the IST-CAR-506 study and on April 30th 2018 for the IST-CAR-561 study. Data were analyzed using R software (version 3.5.1).

\section{Results}

One hundred and twenty-one transplant-ineligible NDMM patients were analyzed: 63 from the IST-CAR-

Table 2. Grade 3-5 treatment-related adverse events during induction and maintenance therapy.

\begin{tabular}{|c|c|c|}
\hline Grade 3-5 AE & $\begin{array}{c}\text { IST-CAR-561 } \\
\text { Once-weekly } \\
\mathrm{N}=63(\%)\end{array}$ & $\begin{array}{c}\text { IST-CAR-506 } \\
\text { Twice-weekly } \\
\text { N=56 (\%) }\end{array}$ \\
\hline At least 1 hematologic $\mathrm{AE}$ & $15(24)$ & $17(30)$ \\
\hline Anemia & $2(3)$ & $6(11)$ \\
\hline Neutropenia & $13(21)$ & $12(21)$ \\
\hline Thrombocytopenia & $4(6)$ & $3(5)$ \\
\hline At least 1 non-hematologic $\mathrm{AE}$ & $24(38)$ & $23(41)$ \\
\hline Cardiac & $4(6)$ & $5(9)$ \\
\hline - Heart failure & $3(5)$ & $2(4)$ \\
\hline - Myocardial infarction & 0 & $1(2)$ \\
\hline - Atrial fibrillation/flutter & 0 & $2(4)$ \\
\hline - Sudden death & $1(2)$ & 0 \\
\hline Vascular & $5(8)$ & $6(11)$ \\
\hline - Hypertension & $4(6)$ & $2(4)$ \\
\hline Gastrointestinal & $3(5)$ & $3(5)$ \\
\hline Infection & $5(8)$ & $3(5)$ \\
\hline Nervous & $2(3)$ & $3(5)$ \\
\hline Respiratory & $4(6)$ & $1(2)$ \\
\hline - Pulmonary edema & $3(5)$ & $1(2)$ \\
\hline Fatigue & 0 & $3(5)$ \\
\hline Creatinine increase & $2(3)$ & 0 \\
\hline At least 1 dose reduction for carfilzomib & $18(29)$ & $17(30)$ \\
\hline Patients who discontinued carfilzomib due to $\mathrm{AE}$ & $17(27)$ & $17(30)$ \\
\hline
\end{tabular}

$\mathrm{AE}$ : adverse events. 
561 study (once-weekly carfilzomib) and 58 from the IST-CAR-506 study (twice-weekly carfilzomib). Patients' characteristics are listed in Table 1 . The median age at diagnosis in the entire population was 72 years (range, 55 86 years). Cytogenetic data were available in 94 patients: 37 (31\%) had high-risk chromosomal abnormalities by FISH, including $10 \%$ of patients with $\mathrm{t}(4 ; 14)$, 3\% with $\mathrm{t}(14 ; 16)$, and $18 \%$ with del(17p), while 57 patients $(47 \%)$ were classified as standard-risk. No significant differences were observed in the two groups between the percentage of patients with ISS 3 disease $(32 \%$ vs. $40 \% ; P=0.45)$, high-risk FISH (30\% vs. $31 \% ; P=0.40)$ or Frailty Score $(6 \%$ vs. $17 \% ; P=0.09)$. The median follow up of the entire cohort was 39 months [interquartile range (IOR): 31-47], without any difference between the two groups.

Overall, 119 of 121 patients enrolled in the studies started induction therapy (Figure 1): 63 in the once-weekly group and 56 in the twice-weekly group. Two patients did not start therapy in the twice-weekly group: one withdrew consent and one was lost to follow up. Ninety patients entered the maintenance phase: 47 (75\%) and 43 $(74 \%)$ in the once- and twice-weekly groups, respectively (Figure 1).

In the ITT population, the median PFS from enrollment was 35.7 months (95\%CI: 23.7-NR) in the once-weekly group and 35.5 months (95\%CI: $24.3-\mathrm{NR}$ ) in the twice- weekly group, with, respectively, $47 \%$ and $49 \%$ of patients alive and free from progression at three years (Figure 2A). When adjusting for age, ISS, FISH, and Frailty Score, no significant differences in the risk of progression or death were observed between the once-weekly and the twice-weekly carfilzomib groups (HR: $1.39 ; P=0.26$ ). Median PFS-2 was similar in patients receiving onceweekly (48.6 months; 95\% CI: 36.5-NR) and twice-weekly (48.5 months; 95\%CI: 44.1-NR) carfilzomib (HR: 1.25; $P=0.51$ ) (Figure $2 \mathrm{~B}$ ). At three years, median $\mathrm{OS}$ was not reached in either group, with $70 \%$ and $72 \%$ of patients alive in the two groups, respectively (Figure 2C). No difference in the risk of death was observed between the once-weekly and the twice-weekly carfilzomib groups when adjusting for age, ISS, FISH and Frailty Score (HR: 1.27; $P=0.50)$. We also assessed PFS and $O S$ according to cytogenetic risk. No significant difference in 3-year PFS (52\% vs. 43\%; HR: $0.76 ; P=0.38)$ and 3 -year OS (78\% vs. $73 \%$; HR: $0.71 ; P=0.36)$ was reported between standardand high-risk FISH patients, with a greater reduction in the risk of progression or death in the once-weekly (HR: 1.17; $P=0.72)$ than in the twice-weekly carfilzomib group (HR: $0.52 ; P=0.12$; interaction $P=0.19$ ).

The median duration of maintenance was 17 months (IOR: 4-28) in the once-weekly and 20 months (IOR 7-32) in the twice-weekly group $(P=0.17)$. At three years, PFS_m
A PFS

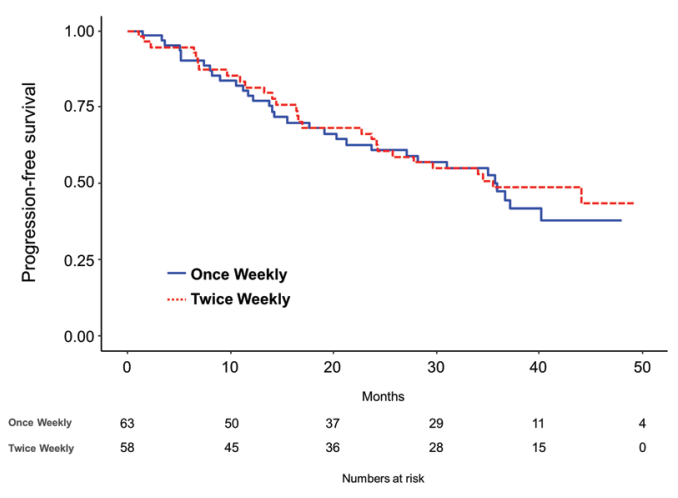

B PFS-2

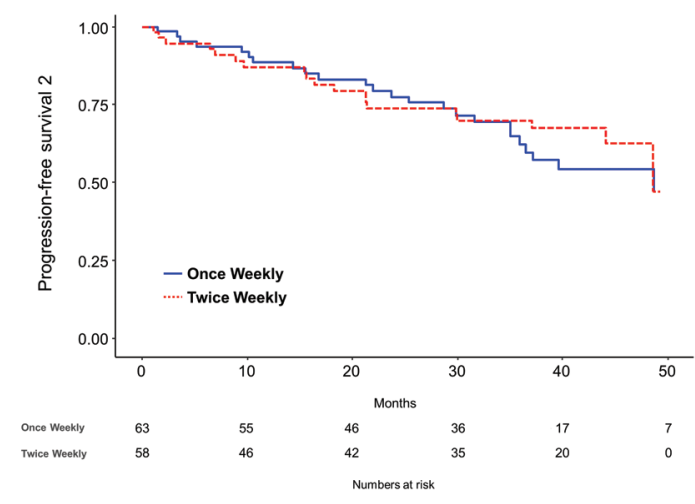

C os

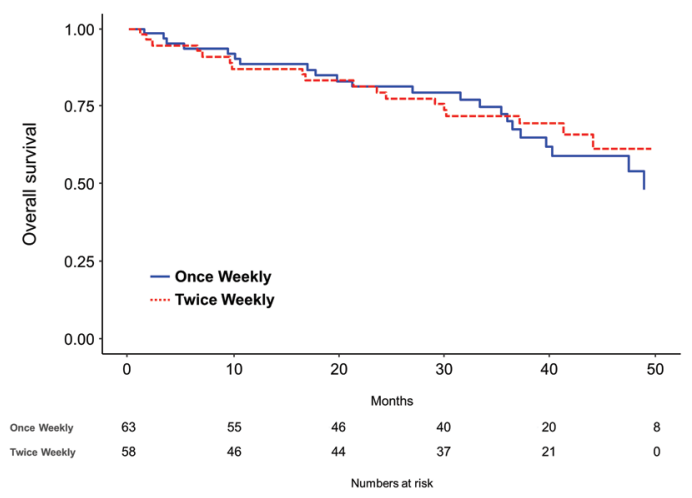

Figure 2. Once-weekly versus twice-weekly carfilzomib in patients with newly diagnosed multiple myeloma. (A) Intention-to-treat progression-free survival (ITT PFS). (B) Intention-to-treat progression-free survival 2 (ITT PFS-2). (C) Intention-to-treat overall survival (ITT OS). (Note that PFS-2 was calculated from the date of enrollment to the date of second relapse/progression or death or the date the patient was last known to be in remission.) 
was $47 \%$ (95\% CI: 33\%-68\%) and 51\% (95\% CI: 38\%$70 \%$ ) in the once-weekly group and in the twice-weekly group, respectively (Figure $3 \mathrm{~A}$ ), with no significant difference in the risk of progression (HR: $1.04 ; P=0.92)$ within the two groups when adjusting for age, ISS, FISH, Frailty Score and response to induction. No differences in 3-year PFS-2_m (65\% vs. 74\%; HR: 0.85; $P=0.74)$ and OS_m (72\% vs. 73\%; HR: 0.82; $P=0.71$ ) were observed between the two groups (Figure $3 \mathrm{~B}$ and $\mathrm{C}$ ).

Overall, the proportion of patients achieving a PR or better was $92 \%$ in the once-weekly versus $90 \%$ in the twice-weekly group ( $P=0.76$ ), including $22 \%$ and $29 \%$ of patients obtaining a $C R$ or better $(P=0.41)$. Responses were rapid: median time to PR or better was 1.9 months in the once-weekly group and 1.2 months in the twiceweekly group.

Carfilzomib dose reduction was necessary in 18 (29\%) patients receiving the once-weekly schedule and in 17 $(30 \%)$ patients receiving the twice-weekly schedule. The median relative dose intensity of carfilzomib [once weekly $97.6 \%$ (IOR 88.3-100\%); twice weekly 97.2\% (IOR $90.4-100 \%)$ ] was similar in the two groups $(P=0.75)$. Dexamethasone dose reduction was necessary in 13 $(21 \%)$ patients receiving the once-weekly schedule and in $18(32 \%)$ patients receiving the twice-weekly schedule. The median relative dose intensity of dexamethasone [once weekly 100\% (IOR 82.6-100\%); twice weekly $100 \%$ (IOR 88.5-100\%)] was similar in the two groups $(P=0.85)$. Cyclophosphamide dose reduction was necessary in $7(11 \%)$ patients receiving the once-weekly schedule and in 15 (27\%) patients receiving the twice-weekly schedule. Nevertheless, the median relative dose intensity of cyclophosphamide [once weekly 96.85\% (IOR 90.8$100 \%$ ); twice weekly 96.75\% (IOR 88.6-100\%)] was similar in the two groups $(P=0.97)$. The most common $\mathrm{AE}$ leading to carfilzomib dose reduction were acute kidney injury (1 patient in the once-weekly group and 2 patients in the twice-weekly group), infections (2 patients in each group), and hypertension (4 patients in the once-weekly group and none in the twice-weekly group). Treatmentrelated $\mathrm{AE}$ leading to the discontinuation of carfilzomib occurred in $17(27 \%)$ patients in the once-weekly group and $17(30 \%)$ patients in the twice-weekly group. The most common AE leading to carfilzomib discontinuation were cardiac injury ( 6 patients in the once-weekly group and 6 patients in the twice-weekly group), infections ( 3 patients in the once-weekly group and 3 patients in the twice-weekly group), and thromboembolism (2 patients in the once-weekly group and 1 in the twice-weekly group). Cardiac events leading to drug discontinuation during induction (3 and 2) and maintenance (3 and 4) occurred at similar rates in patients receiving once- versus

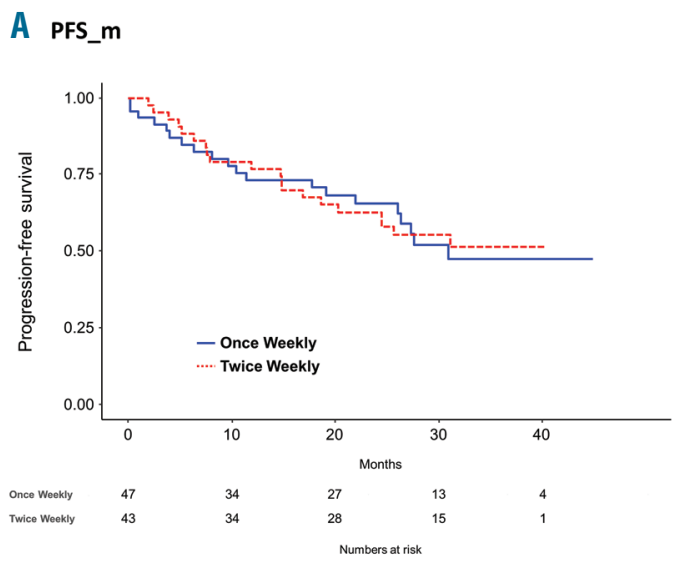

B PFS-2 m

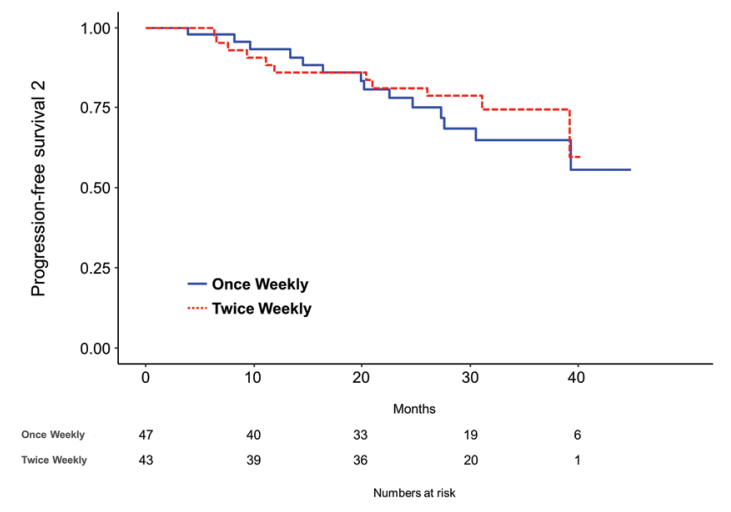

C OS_m

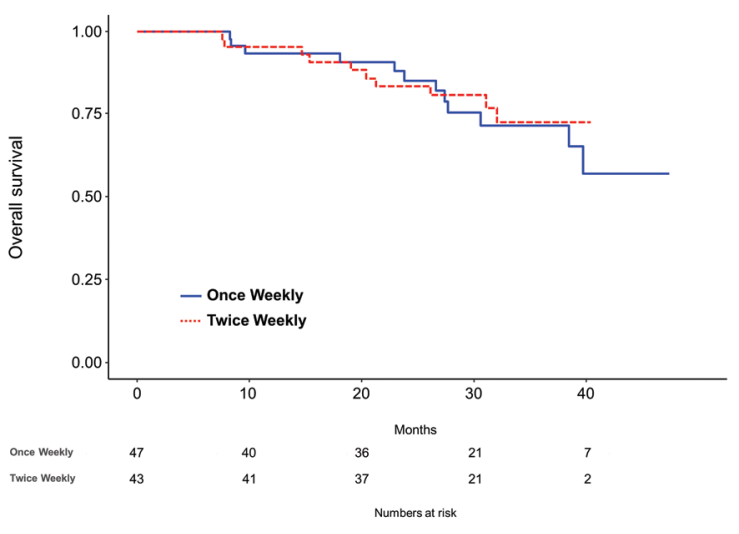

Figure 3. Analysis from start of maintenance therapy. (A) Progression-free survival from start of maintenance therapy (PFS_m). (B) Progression-free survival 2 from start of maintenance therapy (PFS-2_m). (C) Overall survival from start of maintenance therapy (OS_m). (Note that PFS-2 was calculated from the date of enrollment to the date of second relapse/progression or death or the date the patient was last known to be in remission.) 
twice-weekly carfilzomib, respectively. Similarly, the rates of infections leading to drug discontinuation were similar during induction (2 and 3 ) and maintenance (1 and 0 ) between the once-versus twice-weekly groups.

Overall, the incidence of treatment-related grade 3-5 AE was similar in the once-weekly and the twice-weekly carfilzomib groups, both in terms of hematologic (24\% vs. $30 \% ; P=0.82)$ and non-hematologic $(38 \%$ vs. $41 \%$; $P=0.83)$ AE. The most frequent non-hematologic grade $\geq 3$ $\mathrm{AE}$ were infections $[5(8 \%)$ in the once-weekly group vs. $3(5 \%)$ in the twice-weekly group], respiratory [4 (6\%) vs. $1(2 \%)]$, cardiac $[4(6 \%)$ vs. $5(9 \%)$ ] and hypertension [4 $(6 \%)$ vs. $2(4 \%)$ ]. The incidence of treatment-related grade 3-5 AE during carfilzomib maintenance was low, with comparable rates of hematologic $(0 \%$ vs. $5 \%)$ and nonhematologic (21\% vs. $23 \%) \mathrm{AE}$ in the once-weekly and the twice-weekly groups. The most frequent $\geq 3 \mathrm{AE}$ was hypertension [4 $(9 \%)$ vs. none]. All AE are reported in Table 2.

\section{Discussion}

In this pooled analysis of two phase I/II studies comparing two alternative schedules of carfilzomib, transplantineligible NDMM patients who received once-weekly carfilzomib at the dose of $70 \mathrm{mg} / \mathrm{m}^{2}$ showed similar response rates as compared to patients treated with twiceweekly carfilzomib at the dose of $36 \mathrm{mg} / \mathrm{m}^{2}$. Moreover, the analysis did not report differences in terms of PFS, PFS2 , and $O S$. Administering high-dose carfilzomib (70 $\mathrm{mg} / \mathrm{m}^{2}$ ) in a once-weekly schedule did not impair the safety profile of the KCyd combination in comparison with a lower $\left(36 \mathrm{mg} / \mathrm{m}^{2}\right)$ twice-weekly schedule.

To date, two doses of twice-weekly carfilzomib, 27 $\mathrm{mg} / \mathrm{m}^{2}$ and $56 \mathrm{mg} / \mathrm{m}^{2}$, have been approved for the treatment of RRMM patients, based on the results of the phase III ASPIRE and ENDEAVOR trials. In the ASPIRE study, carfilzomib was tested at the dose of $27 \mathrm{mg} / \mathrm{m}^{2}{ }^{13}$ However, a higher dose of carfilzomib $\left(36 \mathrm{mg} / \mathrm{m}^{2}\right)$ had been investigated in combination with lenalidomide and dexamethasone, and was shown to be safe and effective for NDMM patients. ${ }^{4,14-16}$ In the ENDEAVOR trial, which compared $\mathrm{Kd}$ versus bortezomib-dexamethasone $(\mathrm{Vd})$, carfilzomib was administered at the dose of $56 \mathrm{mg} / \mathrm{m}^{2}{ }^{2}$. 18

Despite the great results yielded by the introduction of carfilzomib, treatment compliance and quality of life of young active patients, as well as those of elderly patients with reduced mobility, are compromised by the need for frequent visits to the outpatient clinic for carfilzomib dosing. From this point of view, a shift from the current twice-weekly to a once-weekly dosing schedule would decrease by $50 \%$ patient visits to health care facilities, with a subsequent improvement in quality of life and a reduction in drug and health care costs. For these purposes, higher doses of carfilzomib, administered once-weekly, were tested in the relapse setting in a phase Ib/II study and in a subsequent phase III study.-9 Once-weekly carfilzomib yielded a higher ORR as compared to twice-weekly carfilzomib, resulting in prolonged median PFS (11.2 vs. 7.6 months) without significantly increasing the rate of $\mathrm{AE}$ or the risk of treatment discontinuation due to $\mathrm{AE}$. However, the major limitation of the ARROW study was the low dose adopted for the twice-weekly arm, which was chosen by the investigators because it was the approved dose at the time of trial design. Indeed, higher doses of carfilzomib (up to $36 \mathrm{mg} / \mathrm{m}^{2}$ when given in combination and $70 \mathrm{mg} / \mathrm{m}^{2}$ alone) have been safely delivered both in upfront and relapse settings. ${ }^{5,8,14-20}$

To our knowledge, this is the first analysis to compare two different schedules and doses of carfilzomib (70 $\mathrm{mg} / \mathrm{m}^{2}$ once-weekly vs. $36 \mathrm{mg} / \mathrm{m}^{2}$ twice-weekly) as induction and maintenance therapies for elderly, transplant-ineligible NDMM patients.

In the ITT analysis, we observed no significant differences in 3-year PFS (47\% vs. 49\%), PFS-2 (62\% vs. $70 \%$ ) and $\mathrm{OS}(70 \%$ vs. $72 \%)$ in patients receiving once- versus twice-weekly carfilzomib. The risks of dose reduction or treatment discontinuation were equal between the two groups. Of note, delivering $70 \mathrm{mg} / \mathrm{m}^{2}$ of carfilzomib in a single dose did not increase the risk of grade 3-5 hematologic $(24 \%$ vs. $30 \% ; P=0.82)$ and non-hematologic (38\% vs. $41 \% ; P=0.83) \mathrm{AE}$, as compared to a twice-weekly administration of $36 \mathrm{mg} / \mathrm{m}^{2}$ of carfilzomib. Importantly, no new cardiovascular safety risks were identified with once-weekly carfilzomib treatment at the $70 \mathrm{mg} / \mathrm{m}^{2}$ dose.

The aim of continuous treatment is to prolong PFS and OS among NDMM patients without negatively affecting their quality of life. For this purpose, we compared onceversus twice-weekly maintenance with carfilzomib. Among patients who received carfilzomib maintenance, we did not observe any significant differences in terms of 3-yearPFS_m (47\% vs. 51\%), PFS-2_m (65\% vs. 74\%), and OS_m $(72 \%$ vs. $73 \%)$ between the once-weekly and twice-weekly schedules. Continuous treatment with single-agent carfilzomib was well tolerated and grade 3-5 AE were infrequent in both groups, although patients in the once-weekly arm were at higher risk of developing grade $3-5$ hypertension ( $9 \%$ vs. $0 \%$ ) as compared to patients in the twice-weekly group.

As previously reported, carfilzomib is able to at least partially abrogate the unfavorable prognostic significance of high-risk FISH cytogenetic abnormalities. ${ }^{21}$ In this trial, we observed no difference between standard and highrisk FISH patients in terms of 3 -year PFS (52\% vs. $43 \%$ ) and OS (78\% vs. 73\%), with a greater reduction in the risk of progression or death for high-risk FISH patients (as compared to standard-risk FISH patients) in the onceweekly (HR: 1.17) than in the twice-weekly (HR: 0.52) carfilzomib schedule. These results compared favorably with those observed in the ARROW trial. ${ }^{9}$ However, due to the high frequency of patients with unknown cytogenetic risk and the small number of patients, these results should be interpreted with caution and this evidence must be confirmed by further studies.

The major limitation of our analysis was the non-randomized design of the two phase I/II studies. Indeed, the study populations were slightly different (e.g. frailty status, FISH data availability), but the most important inclusion and exclusion criteria, as well as the treatment schema, were identical. Furthermore, the reproducibility of the results in the community setting was limited both by the lower percentage of older patients ( $\geq 75$ years) as compared to other studies (such as the FIRST and the ALCYONE), and by the fact that patients included in this analysis were treated in the context of clinical trials in a limited number of selected, experienced centers. With this limitation, our results should be interpreted with caution, even though they should be considered as the basis of future randomized trials. 
In conclusion, a once-weekly $70 \mathrm{mg} / \mathrm{m}^{2}$ infusion of carfilzomib was shown to be as safe and effective as a twice weekly $36 \mathrm{mg} / \mathrm{m}^{2}$ infusion for the initial treatment of elderly transplant-ineligible NDMM patients, both as induction therapy in combination with cyclophosphamide and dexamethasone and as single-agent maintenance. This analysis supports the use of high-dose onceweekly carfilzomib and provides the rationale for the investigation of once- versus twice-weekly carfilzomib as initial treatment for $\mathrm{MM}$ patients.

\section{Funding}

The IST-CAR-561 (NCT01857115) study was sponsored by Stichting Hemato-Oncologie voor Volwassenen Nederland (HOVON, the Netherlands), in collaboration with Fondazione
Neoplasie Sangue ONLUS (Italy). The IST-CAR-506 (NCT01346787) study was sponsored by the HOVON Foundation and co-sponsored by Fondazione Neoplasie Sangue ONLUS. Both trials were supported by funding from AMGEN (Onyx Pharmaceuticals), which had no role in study design, data collection, data analysis, data interpretation, writing of the report or publication of this article. The corresponding author had full access to all the data in the two studies and had final responsibility for the decision to prepare and submit this manuscript for publication, together with the other authors.

\section{Acknowledgments}

We thank the patients who participated in these studies and their families; the study co-investigators, nurses, and coordinators at each of the clinical sites.

\section{References}

1. Kumar SK, Dispenzieri A, Lacy MQ, et al. Continued improvement in survival in multiple myeloma: changes in early mortality and outcomes in older patients. Leukemia. 2014;28(5):1122-1128

2. San Miguel JF, Schlag R, Khuageva NK, et al. Persistent Overall Survival Benefit and No Increased Risk of Second Malignancies With Bortezomib-Melphalan-Prednisone Versus Melphalan-Prednisone in Patients With Previously Untreated Multiple Myeloma. J Clin Oncol. 2013;31(4):448455.

3. Bringhen S, Larocca A, Rossi D, et al. Efficacy and safety of once-weekly bortezomib in multiple myeloma patients. Blood. 2010;116(23):4745-4753.

4. Dytfeld D, Jasielec J, Griffith KA, et al. Carfilzomib, lenalidomide, and low-dose dexamethasone in elderly patients with newly diagnosed multiple myeloma. Haematologica. 2014;99(9):e162-164.

5. Facon T, Lee JH, Moreau P, et al. Phase 3 Study (CLARION) of Carfilzomib, Melphalan, Prednisone (KMP) V Bortezomib, Melphalan, Prednisone (VMP) in Newly Diagnosed Multiple Myeloma (NDMM). Clin Lymphoma Myeloma Leuk. 2017;17(1):e26-e27.

6. Bringhen S, Petrucci MT, Larocca A, et al. Carfilzomib, cyclophosphamide, and dexamethasone in patients with newly diagnosed multiple myeloma: a multicenter, phase 2 study. Blood. 2014;124(1):63-69.

7. Bringhen S, D'Agostino M, De Paoli L, et al. Phase $1 / 2$ study of weekly carfilzomib, cyclophosphamide, dexamethasone in newly diagnosed transplant-ineligible myeloma. Leukemia. 2018;32(4):979-985.

8. Berenson JR, Cartmell A, Bessudo A, et al.
CHAMPION-1: a phase $1 / 2$ study of onceweekly carfilzomib and dexamethasone for relapsed or refractory multiple myeloma. Blood. 2016;127(26):3360-3368.

9. Moreau P, Mateos M-V, Berenson JR, et al. Once weekly versus twice weekly carfilzomib dosing in patients with relapsed and refractory multiple myeloma (A.R.R.O.W.) interim analysis results of a randomised phase 3 study. Lancet Oncol. 2018; 19(7):953-964.

10. National Cancer Institute [USA]. Common Terminology Criteria for Adverse Events (CTCAE) Version. 4.0. US Department of Health and Human Services. May 28, 2009.

11. Sonneveld P, Avet-Loiseau H, Lonial S, et al. Treatment of multiple myeloma with highrisk cytogenetics: a consensus of the International Myeloma Working Group. Blood. 2016;127(24):2955-2962.

12. Palumbo A, Bringhen S, Mateos M-V, et al. Geriatric assessment predicts survival and toxicities in elderly myeloma patients: An International Myeloma Working Group report. Blood. 2015;125(13):2068-2074

13. Stewart AK, Rajkumar SV, Dimopoulos MA, et al. Carfilzomib, lenalidomide, and dexamethasone for relapsed multiple myeloma. $\mathrm{N}$ Engl J Med. 2015;372(2):142-152.

14. Jakubowiak AJ, Dytfeld D, Griffith KA, et al. A phase $1 / 2$ study of carfilzomib in combination with lenalidomide and low-dose dexamethasone as a frontline treatment for multiple myeloma. Blood. 2012; 120(9):1801-1809.

15. Gay F, Rota Scalabrini D, Belotti A, et al. Updated efficacy and MRD data according to risk-status in newly diagnosed myeloma patients treated with carfilzomib plus lenalidomide or cyclosphosphamide: Results from the FORTE trial. HemaSphere 2018;2(S1):6. [Abstract \#S109, EHA 2018 $23^{\text {rd }}$ Congress].

16. Gay F, Foà R, Musto $\mathrm{P}$, et al. Updated efficacy data and MRD analysis according to risk status in newly diagnosed myeloma patients treated with carfilzomib + lenalidomide or cyclophosphamide (FORTE trial). J Clin Oncol. 2018;36(15_suppl) [Abstract \#8009, ASCO 2018 Annual Meeting].

17. Dimopoulos MA, Moreau P, Palumbo A, et al. Carfilzomib and dexamethasone versus bortezomib and dexamethasone for patients with relapsed or refractory multiple myeloma (ENDEAVOR): a randomised, phase 3 , open-label, multicentre study. Lancet Oncol. 2016;17(1):27-38.

18. Dimopoulos MA, Goldschmidt $\mathrm{H}$ Niesvizky R, et al. Carfilzomib or bortezomib in relapsed or refractory multiple myeloma (ENDEAVOR): an interim overall survival analysis of an open-label, randomised, phase 3 trial. Lancet Oncol. 2017;18(10):1327-1337.

19. Boccia RV, Bessudo A, Agajanian R, et al. A Multicenter, Open-Label, Phase 1b Study of Carfilzomib, Cyclophosphamide, and Dexamethasone in Newly Diagnosed Multiple Myeloma Patients (CHAMPION 2). Clin Lymphoma Myeloma Leuk. 2017; 17(7):433-437.

20. Sonneveld P, Asselbergs E, Zweegman S, et al. Phase 2 study of carfilzomib, thalidomide, and dexamethasone as induction/consolidation therapy for newly diagnosed multiple myeloma. Blood. 2015; 125(3):449-456

21. Chng W-J, Goldschmidt H, Dimopoulos $\mathrm{MA}$, et al. Carfilzomib-dexamethasone vs bortezomib-dexamethasone in relapsed or refractory multiple myeloma by cytogenetic risk in the phase 3 study ENDEAVOR. Leukemia. 2017;31(6):1368-1374. 